\title{
An Approach to the Imine Ring System of Pinnatoxins
}

Armen Zakarian* and Matthew J. Pelc

\section{Supporting Information}

Experimental procedures, spectroscopic and analytical data for compounds 1-18.

General Information. All reactions were carried out under an inert atmosphere of dry argon in oven or flame-dried glassware. Proton magnetic resonance spectra were recorded at 300, 400, and $500 \mathrm{MHz}$ on Varian Mercury, Varian Unity Inova, and Varian VXR spectrometers, respectively. Carbon magnetic resonance spectra were recorded at $75 \mathrm{MHz}$ on a Varian Mercury spectrometer. All chemical shifts were reported in $\delta$ units relative to tetramethylsilane. Optical rotations were measured on a PerkinElmer 341 Polarimeter. Infrared spectra were recorded on Perkin-Elmer Paragon 1000 FTIR spectrometer. Elemental analyses were performed by Atlantic Microlab, Inc. (Norcross, GA). High resolution mass spectral data were obtained by the Mass Spectrometry laboratory at Florida State University. Melting points were determined on a 50-600 hot stage controller. Analytical thin-layer chromatography (TLC) was performed using pre-coated TLC plates with silica Gel 60 F254 (E. Merck no. 57157). Flash column chromatography was performed using 40-63 um silica gel (Merck, Geduran, no. 11567-1) as the stationary phase. Tetrahydrofuran (THF) and ether $\left(\mathrm{Et}_{2} \mathrm{O}\right)$ were dried by distilling from sodium-benzophenone in a continuous still under an atmosphere of argon. Dichloromethane, di-iso-propylamine, pyridine, triethylamine, and chlorotrimethylsilane were distilled from calcium hydride in a continuous still under an atmosphere of argon. Chlorotriethylsilane (TESCl), and di-iso-propylethyl amine (Hunig's base) were distilled from calcium hydride under an inert atmosphere of dry argon and stored over calcium hydride. 1,3-Dimethyl3, 4, 5,6-tetrahydro-2(1H)-pyrimidinone (DMPU) and dimethyl sulfoxide (DMSO) were distilled under reduced pressure from calcium hydride and stored over calcium hydride.

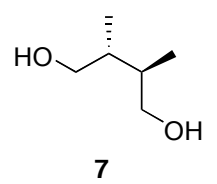

Diol 7. To a solution of bis-imide $\mathbf{6}^{1}(4.55 \mathrm{~g}, 12.3 \mathrm{mmol})$ in $\mathrm{THF}$ (62 ml) and water $(49 \mathrm{ml})$ was added $\mathrm{LiOH} \cdot \mathrm{H}_{2} \mathrm{O}(2.06 \mathrm{~g}, 49.0 \mathrm{mmol})$. The mixture was $\mathrm{cooled}$ to $0{ }^{\circ} \mathrm{C}$ and $30 \%$ aqueous hydrogen peroxide (12.3 $\mathrm{ml})$ was added dropwise. The resulting mixture was stirred at room temperature for $18 \mathrm{~h}$, and then quenched carefully with $1.5 \mathrm{M}$ aqueous $\mathrm{Na}_{2} \mathrm{SO}_{3}$. The aqueous layer was extracted with dichloromethane (3x50 ml), acidified with conc. aqueous $\mathrm{HCl}(25 \mathrm{ml})$, and extracted with EtOAc (5x50 ml). The ethyl acetate extracts were dried with anhydrous $\mathrm{Na}_{2} \mathrm{SO}_{4}$ and concentrated under reduced pressure to give $1.86 \mathrm{~g}(12.73 \mathrm{mmol}$, 98\%) of 2R, 3R-dimethylsuccinic acid that was used without further purification. ${ }^{1} \mathrm{H}$ NMR (300 MHz, DMSO); $\delta(\mathrm{ppm})$ : 12.10 (s, $\left.2 \mathrm{H}\right)$; $2.58(\mathrm{~m}, 2 \mathrm{H}) ; 1.2(\mathrm{~d}, \mathrm{~J}=7.2 \mathrm{~Hz}, 6 \mathrm{H})$.

A solution of the diacid $(1.75 \mathrm{~g}$, $12.0 \mathrm{mmol})$ in dry THF (100 ml) was treated with

\footnotetext{
${ }^{1}$ Kise, N.; Ueda, T.; Kumada, K.; Terao, Y.; Ueda, N. J. Org. Chem. 2000, 65, 464-468.
} 
lithium aluminum hydride $(1.6 \mathrm{~g}, 42 \mathrm{mmol})$ at reflux for $12 \mathrm{~h}$. After cooling to $0^{\circ} \mathrm{C}$, the mixture was diluted with ether and $1.6 \mathrm{ml}$ of water, $1.6 \mathrm{ml}$ of $3 \mathrm{M}$ aqueous $\mathrm{NaOH}$, and $4.8 \mathrm{ml}$ of water were added dropwise and the mixture was stirred for $1 \mathrm{~h}$ at room temperature. The white precipitate was filtered off and the solution was concentrated under reduced pressure to give, after drying in vacuum, $1.42 \mathrm{~g}$ (12 mmol, 100\%) of the crude diol, ${ }^{2}$ which was used in the next step without further purification. $[\alpha]_{\mathrm{D}}{ }^{20}+8.0^{\circ}\left(\mathrm{C} 1.0 \mathrm{CH}_{2} \mathrm{Cl}_{2}\right)$, lit. +5.2 ( C 1.7, $\left.\mathrm{Et} \mathrm{t}_{2} \mathrm{O}\right) .{ }^{3}{ }^{1} \mathrm{H} \mathrm{NMR}(300 \mathrm{MHz}$, $\left.\mathrm{CDCl}_{3}\right) ; \delta(\mathrm{ppm}): 3.60(\mathrm{dd}, \mathrm{J} 1=10.8 \mathrm{~Hz}, \mathrm{~J} 2=4.2 \mathrm{~Hz}, 2 \mathrm{H}) ; 3.48(\mathrm{dd}, \mathrm{J} 1=10.8 \mathrm{~Hz}, \mathrm{~J} 2=6.0$ $\mathrm{Hz}, 2 \mathrm{H}) ; 3.29(\mathrm{bs}, 2 \mathrm{H}) ; 1.70(\mathrm{~m}, 2 \mathrm{H}) ; 0.88(\mathrm{~d}, \mathrm{~J}=7.2 \mathrm{~Hz}, 6 \mathrm{H})$.



4

Iodide 4. The diol $(3.08 \mathrm{~g}, 26.10 \mathrm{mmol})$ was added to a susupesion of sodium hydride (1.25 g of $60 \%$ in oil, $31.32 \mathrm{mmol}$, then washed from mineral oil) in $\mathrm{THF}$ at $0^{\circ} \mathrm{C}$. After $30 \mathrm{~min}$ at room temperature, pivaloyl chloride $(3.84 \mathrm{~mL}, 31.32 \mathrm{mmol})$ was added dropwise at $0^{\circ} \mathrm{C}$. The reaction mixture was stirred at room temperature for $1 \mathrm{~h}$, then quenched with saturated aqueous ammonium chloride, extracted with ethyl acetate and the combined organic layers were washed with brine, dried with anhydrous sodium sulfate, and concentrated under reduced pressure. The resultant oil was purified by column chromatography (silica, 30\% ethyl acetate-hexanes) to give 4.129 (20.38 mmol, 78\%) of the pivaloate. $[\alpha]_{\mathrm{D}}^{20}-4.5^{\circ}\left(\mathrm{c} 1.0 \mathrm{CH}_{2} \mathrm{Cl}_{2}\right)$. IR (film, $\left.\mathrm{cm}^{-1}\right): 3442,2968$, 2934, 1730, 1712, 1480, 1462, 1286, 1162, 1034. ${ }^{1} \mathrm{H} \operatorname{NMR}\left(300 \mathrm{MHz}, \mathrm{CDCl}_{3}\right) ; \delta(\mathrm{ppm}): 3.96$ $(\mathrm{AB}, 2 \mathrm{H}) ; 3.56(\mathrm{dd}, \mathrm{J} 1=6.6 \mathrm{~Hz}, \mathrm{~J} 2=4.2 \mathrm{~Hz}, 1 \mathrm{H}) ; 3.51(\mathrm{dd}, \mathrm{J} 1=6.6 \mathrm{~Hz}, \mathrm{~J} 2=4.2 \mathrm{~Hz}, 1 \mathrm{H})$; $2.03(\mathrm{~m}, 1 \mathrm{H}) ; 1.75(\mathrm{~m}, 1 \mathrm{H}) ; 1.21(\mathrm{~s}, 9 \mathrm{H}) ; 0.88(\mathrm{~d}, \mathrm{~J}=7.2 \mathrm{~Hz}, 6 \mathrm{H}) .{ }^{13} \mathrm{C} \operatorname{NMR}(75 \mathrm{MHz}$, $\left.\mathrm{CDCl}_{3}\right) ; \delta(\mathrm{ppm}): 178.8,68.0,66.3,39.0,37.2,33.5,27.4,12.4,12.3$.

Iodine $(0.17 \mathrm{~g}, 0.69 \mathrm{mmol})$ was added to a solution of the pivaloate $(0.12 \mathrm{~g}, 0.53$ mmol), triphenylphosphine $(0.18 \mathrm{~g}, 0.69 \mathrm{mmol})$, and imidazole $(0.036 \mathrm{~g}, 0.53 \mathrm{mmol})$ in dry dichloromethane $(2 \mathrm{ml})$. The mixture was stirred at room temperature for 14 $\mathrm{h}$, then washed with $1 \mathrm{M}$ aqueous $\mathrm{HCl}$ and water, and the combined aqueous layers were extracted with dichloromethane. The combined organic layers were washed with saturated aqueous $\mathrm{NaHCO}_{3}$, dried with anhydrous sodium sulfate, and concentrated under reduced pressure. The resultant oil was purified by column chromatography (silica, 20\% ethyl acetate-hexanes) to give $0.158 \mathrm{~g}(0.51 \mathrm{mmol}$, 95\%) of the title iodide. $[\alpha]_{\mathrm{D}}{ }^{20}-5.1^{\circ}\left(\mathrm{C} 1.0 \mathrm{CH}_{2} \mathrm{Cl}_{2}\right)$. IR (film, $\left.\mathrm{cm}^{-1}\right): 2968,2934,2360,1730,1480$,

\footnotetext{
${ }^{2}$ McCasland, G. E.; Proscow, S. J. Am. Chem. Soc. 1956, 78, 5646.

${ }^{3}$ Ghosez, L.; Mahuteau-Betzer, F.; Genicot, C.; Vallibera, A.; Cordier, J.-F. Chem. Eur. J. 2002, 8, 3411.
} 
1456, 1284, 1154, 1034. ${ }^{1} \mathrm{H} \mathrm{NMR}$ (300 $\left.\mathrm{MHz}, \mathrm{CDCl}_{3}\right) ; \delta(\mathrm{ppm}): 3.96$ (AB, 2H); 3.22 (dd, $\mathrm{J} 1=9.8 \mathrm{~Hz}, \mathrm{~J} 2=6.0 \mathrm{~Hz}, 1 \mathrm{H}) ; 3.18(\mathrm{dd}, \mathrm{J} 1=9.8 \mathrm{~Hz}, \mathrm{~J} 2=6.6 \mathrm{~Hz}, 1 \mathrm{H}) ; 2.03$ (m, $1 \mathrm{H}) ; 1.75$ $(\mathrm{m}, 1 \mathrm{H}) ; 1.21(\mathrm{~s}, 9 \mathrm{H}) ; 0.98(\mathrm{~d}, \mathrm{~J}=6.6 \mathrm{~Hz}, 3 \mathrm{H}) ; 0.88(\mathrm{~d}, \mathrm{~J}=7.2 \mathrm{~Hz}, 3 \mathrm{H}) .{ }^{13} \mathrm{C} \mathrm{NMR}(75$ $\left.\mathrm{MHz}, \mathrm{CDCl}_{3}\right) ; \delta(\mathrm{ppm}): 178.4,67.1,38.9,36.6,36.2,27.2,16.1,14.1,11.9$.

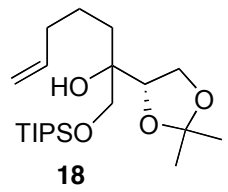

Alcohol 18. A solution of 4-pentenylmagnesium bromide (1.0M in THF, $9.80 \mathrm{ml}, 9.80$ mmol) was added dropwise to a solution of $\mathbf{8}^{4}$ (2.17 $\left.\mathrm{g}, 6.86 \mathrm{mmol}\right)$ in dry THF (14 mL) under argon at $-78^{\circ} \mathrm{C}$. The solution was allowed to stir for 1 hr and then allowed to warm to room temperature. The solution was quenched with aqueous ammonium chloride, and the mixture was extracted with ethyl acetate. The combined organic layers were then washed with water and brine, and the solution dried with anhydrous sodium sulfate and concentrated under reduced pressure. The resultant crude oil was purified by column chromatography (silica, 7.5\% ethyl acetate-hexanes) to give 2.48 9 (6.42 mmol, 93\%) of an 8:1 inseparable mixture of the diastereomeric addition products. ${ }^{1} \mathrm{H} \operatorname{NMR}\left(300 \mathrm{MHz}, \mathrm{CDCl}_{3}\right) ; \delta(\mathrm{ppm}): 5.87-5.74$ (m, 1H); 5.05-4.92 (m, 2H); $4.25-4.16(\mathrm{~m}, 1 \mathrm{H}) ; 4.03-3.87(\mathrm{~m}, 2 \mathrm{H}) ; 3.72-3.55(\mathrm{~m}, 2 \mathrm{H}) ; 2.38$ ( $\mathrm{s}, 1 \mathrm{H}) ; 2.12-1.97$ $(\mathrm{m}, 2 \mathrm{H}) ; 1.62-1.20(\mathrm{~m}, 10 \mathrm{H}) ; 1.97-1.02(\mathrm{~m}, 21 \mathrm{H})$.

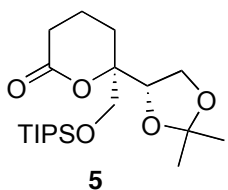

Lactone 5. $\mathrm{OSO}_{4}(0.043 \mathrm{M}$ in $t-\mathrm{BuOH}, 1.9 \mathrm{~mL}, 0.082 \mathrm{mmol})$ was added to a solution of $18(0.46 \mathrm{~g}, 1.19 \mathrm{mmol})$ in diethyl ether (11 mL) and aqueous sodium bicarbonate (2.2 $\mathrm{mL}$ ) which was allowed to stir for 5 minutes. Finely ground sodium periodate (1.27 9, 5.94 mmol) was then added, and the solution stirred vigerously for 8 hours. The solution was then decanted and washed with $\mathrm{Na}_{2} \mathrm{~S}_{2} \mathrm{O}_{3}$, water, brine, and subsequently extracted with ethyl acetate. The organic solution was dried with anhydrous sodium sulfate and concentrated under reduced pressure to afford $1.93 \mathrm{~g}$ the crude oil which was used in the next step without further purification. TPAP (2.3 mg, 0.01 mmol) was added in one portion to a stirred mixture of substrate, NMo(0.25g, 2.13mmol), and powdered $4 \AA$ molecular sieves in dry dichloromethane (10 mL) at r.t. under argon. The reaction was stirred for 3 hr at which time the reaction mixture

\footnotetext{
${ }^{4}$ Marco, A,; Carda, M.; Gonzalez, F.; Rodriguez, S.; Castillo, E.; Murga, J. J. Org. Chem. 1998, 63, 698-707.
} 
was diluted with ethyl acetate and filtered though a 1 cm. pad of silica. The mixture was eluted with dichloromethane and concentrated under reduced pressure. The resultant crude oil was purified by column chromatography (silica, 15\% ethyl acetate-hexanes) to give $0.314 \mathrm{~g}(0.81 \mathrm{mmol}$, 68\% 2-steps) of the major diastereomer 5 and $40 \mathrm{mg}\left(0.103 \mathrm{mmol}\right.$, 9\% over 2 steps) of the minor diatereomer. $5:[\boldsymbol{\alpha}]_{\mathrm{D}}^{20}-\mathbf{1 8}^{\circ} \mathbf{9}^{\circ}$ (c $\left.1.0 \mathrm{CH}_{2} \mathrm{Cl}_{2}\right)$. IR (film, $\mathrm{cm}^{-1}$ ) : 2892, 1746, 1464, 1254, 1216, 1154, 1136, 684. ${ }^{1} \mathrm{H}$ $\operatorname{NMR}\left(300 \mathrm{MHz}, \mathrm{CDCl}_{3}\right) ; \delta(\mathrm{ppm}): 4.18(\mathrm{dd}, \mathrm{J} 1=\mathrm{J} 2=5.1 \mathrm{~Hz}, 1 \mathrm{H}) ; 4.08-4.00$ (m, 2H); 3.72 $(\mathrm{d}, \mathrm{J} 1=7.5 \mathrm{~Hz}, 1 \mathrm{H}) ; 3.67(\mathrm{~d}, \mathrm{~J}=7.5 \mathrm{~Hz}, 1 \mathrm{H}) ; 2.60-2.39$ (m, 2H); 2.08-1.80 (m, 4H) ; $1.38(\mathrm{~s}, 3 \mathrm{H}) ; 1.36(\mathrm{~s}, 3 \mathrm{H}) ; 1.10-1.02(\mathrm{~m}, 21 \mathrm{H}) .{ }^{13} \mathrm{C} \mathrm{NMR}\left(75 \mathrm{MHz}, \mathrm{C}_{6} \mathrm{D}_{6}\right) ; \delta(\mathrm{ppm})$ : $171.3,109.8,83.6,79.1,67.4,64.6,30.6,26.2,26.1,25.9,18.1,17.3,12.1$. HRMS (ESI) calcd for $\mathrm{C}_{20} \mathrm{H}_{38} \mathrm{O}_{5} \mathrm{SiNa}[\mathrm{M}+\mathrm{Na}] 409.23862$, found 409.23903.

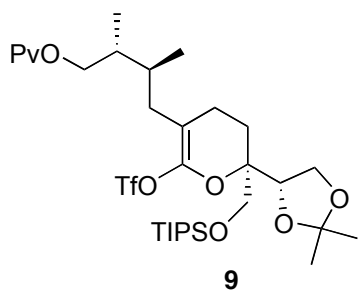

Triflate 9. A solution of the lactone 5 (0.519 g, $1.34 \mathrm{mmol})$ in dry $\mathrm{THF}$ (2.0 $\mathrm{ml}$ total with rinses) was added dropwise to a solution of LDA [1.68 mmol from i-Pr ${ }_{2} \mathrm{NH}$ $(0.26 \mathrm{ml}, 1.85 \mathrm{mmol}), n$-BuLi (2.5M in hexanes, $0.67 \mathrm{mml}, 1.68 \mathrm{mmol}), \mathrm{THF}$ (1.5 ml), $\left.0^{\circ} \mathrm{C}, 10 \mathrm{~min}\right]$ in $\mathrm{THF}$ at $-78^{\circ} \mathrm{C}$ under argon. After $20 \mathrm{~min}$, diethylzinc $(0.18 \mathrm{ml}, 1.76$ mmol) was added, followed by a solution of the iodide 4 (0.59 $\mathrm{g}$, $1.88 \mathrm{mmol})$ in dry $\operatorname{THF}(1.0 \mathrm{ml})$, and then dry DMPU $(1.5 \mathrm{ml})$. The reaction mixture was brought to $0^{\circ} \mathrm{C}$ and stirred for $3 \mathrm{~h}$. A saturated solution of ammonium chloride was added carefully, and then the mixture was extracted with ethyl acetate, and the combined organic layers were washed with water and brine. The solution was dried with anhydrous sodium sulfate and concentrated under reduced pressure. The resultant oil was purified by column chromatography (silica, 15\% ethyl acetate-hexanes) to give 0.651 9 (1.14 mmol, 85\%) of a 5:1 inseparable mixture of the diastereomeric alkylation products. ${ }^{1} \mathrm{H} \operatorname{NMR}\left(500 \mathrm{MHz}, \mathrm{CDCl}_{3}\right)$ for the 5:1 mixture; $\delta(\mathrm{ppm}): 4.19-4.14$ (m, 1H); $4.08-3.99(\mathrm{~m}, 2 \mathrm{H}) ; 3.97-3.88(\mathrm{~m}, 2 \mathrm{H}) ; 2.43-2.30(\mathrm{~m}, 1 \mathrm{H}) ; 2.19-2.10(\mathrm{~m}, 1 \mathrm{H}) ; 2.07-$ $1.74(\mathrm{~m}, 6 \mathrm{H}) ; 1.36(\mathrm{~s}, 6 \mathrm{H}) ; 1.32-1.24(\mathrm{~m}, 1 \mathrm{H}) ; 1.19(\mathrm{~s}, 9 \mathrm{H}) ; 1.10-1.02(\mathrm{~m}, 21 \mathrm{H})$; $0.87(\mathrm{~d}, \mathrm{~J}=6.5 \mathrm{~Hz}, 0.5 \mathrm{H}) ; 0.85(\mathrm{~m}, 5.5 \mathrm{H})$.

A solution of the alkylation product (0.618 g, 1.08 mmol) and N-phenyltriflimide $(0.55 \mathrm{~g}, 1.53 \mathrm{mmol})$ in dry THF $(20 \mathrm{ml})$ was added to a solution of KHMDS (0.5M in PhMe, $6.7 \mathrm{ml}, 3.35 \mathrm{mmol}$ ) during $50 \mathrm{~min}$ using a syringe pump at $-78^{\circ} \mathrm{C}$ under argon. The reaction mixture was stirred for additional 5 min and then quenched by a slow addition of acetic acid $(0.21 \mathrm{ml}, 3.5 \mathrm{mmol})$ at $-78^{\circ} \mathrm{C}$. The reaction mixture was 
brought to room temperature, saturated aqueous ammonium chloride was added, and the mixture was extracted with EtOAc, washed with water and brine, dried with anhydrous sodium sulfate, and concentrated under reduced pressure. The residue was purified by column chromatography (silica, 7\% ethyl acetate-hexanes) to give $0.718 \mathrm{~g}$ (1.02 mmol, 95\%) of the triflate. $[\alpha]_{\mathrm{D}}^{20}-13.7^{\circ}\left(\mathrm{c} 1.0 \quad \mathrm{CH}_{2} \mathrm{Cl}_{2}\right)$. IR $\left(\mathrm{film}, \mathrm{cm}^{-1}\right):{ }^{1} \mathrm{H} \quad \mathrm{NMR}$ $\left(500 \mathrm{MHz}, \mathrm{CDCl}_{3}\right) ; \delta(\mathrm{ppm}): 4.38(\mathrm{dd}, \mathrm{J} 1=\mathrm{J} 2=7.0 \mathrm{~Hz}, 1 \mathrm{H}) ; 4.05-3.99$ (m, 2H); $3.96(\mathrm{dd}$, $\mathrm{J} 1=11.5 \mathrm{~Hz}, \mathrm{~J} 2=7.0 \mathrm{~Hz}, 1 \mathrm{H}) ; 3.90$ (dd, J1=11.5 Hz, J2=6.0 Hz, $1 \mathrm{H}) ; 3.71$ (d, J=11.0 $\mathrm{Hz}, 1 \mathrm{H}) ; 3.67(\mathrm{~d}, \mathrm{~J}=11.0 \mathrm{~Hz}, 1 \mathrm{H}) ; 2.16-2.08$ (m, $3 \mathrm{H}) ; 2.01-1.94$ (m, 2H); 1.89 (ddd, $\mathrm{J} 1=13.9 \mathrm{~Hz}, \mathrm{~J} 2=\mathrm{J} 3=4.6 \mathrm{~Hz}, 1 \mathrm{H}) ; 1.84-1.76(\mathrm{~m}, 2 \mathrm{H}) ; 1.39(\mathrm{~s}, 3 \mathrm{H}) ; 1.35(\mathrm{~s}, 3 \mathrm{H}): 1.20$ $(\mathrm{s}, 9 \mathrm{H}) ; 1.09-1.02(\mathrm{~m}, 21 \mathrm{H}) ; 0.84(\mathrm{~d}, \mathrm{~J}=7.5 \mathrm{~Hz}, 3 \mathrm{H}) ; 0.81(\mathrm{~d}, \mathrm{~J}=6.5 \mathrm{~Hz}, 3 \mathrm{H}) .{ }^{13} \mathrm{C} \mathrm{NMR}$ $\left(75 \mathrm{MHz}, \mathrm{CDCl}_{3}\right) ; \delta(\mathrm{ppm}): 178.4,144.7,118.4(\mathrm{q}, \mathrm{J}=320 \mathrm{~Hz}), 109.3,97.6,82.7,67.6$, $64.3,62.9,38.8,35.5,34.2,31.5,27.2,26.0,25.2,23.0,21.5,17.8,14.1,11.9$, 11.1. HRMS (ESI) calcd for $\mathrm{C}_{32} \mathrm{H}_{57} \mathrm{~F}_{3} \mathrm{O}_{9} \mathrm{SiNa}[\mathrm{M}+\mathrm{Na}]$ 725.33423, found 725.33534 .

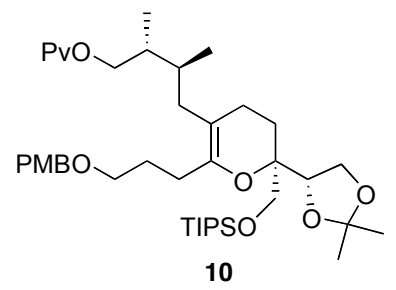

Dihydropyran 10. A solution of 3-(p-methoxybenzyloxy)-propyliodide (0.743 g, 2.43 mmol) in THF (3.5 ml) was added to a solution of t-BuLi (1.5M in pentane, $3.40 \mathrm{ml}$, $5.1 \mathrm{mmol})$ in $\operatorname{THF}(7.0 \mathrm{ml})$ dropwise at $-78^{\circ} \mathrm{C}$. After $10 \mathrm{~min}, \mathrm{ZnCl}_{2}(1.0 \mathrm{M}$ in $\mathrm{THF}, 2.4$ ml, $2.40 \mathrm{mmol}$ ) was added, and the mixture was stirred $5 \mathrm{~min}$. A solution of triflate $9(0.683 \mathrm{~g}, 0.972 \mathrm{mmol})$ in $\operatorname{THF}(3.0 \mathrm{ml}$ with rinses $)$ was added to the alkylzinc reagent followed by freshly distilled NMP (8.0 ml) and Pd[P(Bu-t) 3$]_{2}(25 \mathrm{mg}, 0.049$ mol), and the mixture was warmed to $30^{\circ} \mathrm{C}$ for $30 \mathrm{~min}$. After cooling to room temperature, the reaction mixture was diluted with $30 \%$ EtoAc-hexanes and quenched with saturated aqueous ammonium chloride. The organic phase was washed with water $(3 \times 10 \mathrm{ml})$, the combined aqueous layers were extracted with $30 \%$ EtoAc-hexanes, and the combined organic layers were washed with brine, dried with anhydrous sodium sulfate, and concentrated under reduced pressure. The residue was purified by column chromatography (silica, 5\% ethyl acetate-hexanes with $0.3 \%$ Et ${ }_{3} \mathrm{~N}$ ) to give $0.535 \mathrm{~g}(0.73 \mathrm{mmol}, 75 \%)$ of the coupling product. $[\alpha]_{\mathrm{D}}^{20}-8.9^{\circ}\left(\mathrm{C}^{2.0} \mathrm{CH}_{2} \mathrm{Cl} l_{2}\right) \cdot \mathrm{IR}$ (film, $\left.\mathrm{cm}^{-1}\right): 3442,2956,2924,2874,1614,1514,1462,1380,1372,1248,1210$, $1174,1154,1070,1036,1014 .{ }^{1} \mathrm{H} \mathrm{NMR}\left(500 \mathrm{MHz}, \mathrm{C}_{6} \mathrm{D}_{6}\right) ; \delta(\mathrm{ppm}): 7.26$ (d, J=8.5 Hz, $\left.2 \mathrm{H}\right)$; $6.83(\mathrm{~d}, \mathrm{~J}=8.5 \mathrm{~Hz}, 2 \mathrm{H}) ; 4.62(\mathrm{dd}, \mathrm{J} 1=\mathrm{J} 2=7.5 \mathrm{~Hz}, 1 \mathrm{H}) ; 4.37$ ( $\mathrm{s}, 2 \mathrm{H}) ; 4.26$ ( dd, $\mathrm{J} 1=\mathrm{J} 2=8.5 \mathrm{~Hz}, 1 \mathrm{H}) ; 4.12(\mathrm{dd}, \mathrm{J} 1=8.0 \mathrm{~Hz}, \mathrm{~J} 2=7.0 \mathrm{~Hz}, 1 \mathrm{H}) ; 4.03$ ( dd, J1=11.0 Hz, $\mathrm{J} 2=7.0 \mathrm{~Hz}, 1 \mathrm{H}) ; 3.97(\mathrm{dd}, \mathrm{J} 1=11.0 \mathrm{~Hz}, \mathrm{~J} 2=6.5 \mathrm{~Hz}, 1 \mathrm{H}) ; 3.83(\mathrm{~d}, \mathrm{~J}=10.5 \mathrm{~Hz}$, $1 \mathrm{H}) ; 3.76$ 
$(d, J=10.5 \mathrm{~Hz}, 1 \mathrm{H}) ; 3.45$ (t, J=6.0 Hz, 2H); 3.33 ( $\mathrm{s}, 3 \mathrm{H}) ; 2.45$ (ddd, J1=16.0 Hz, $\mathrm{J} 2=\mathrm{J} 3=8.0 \mathrm{~Hz}, 1 \mathrm{H}) ; 4.37$ (ddd, J1=16.0 Hz，J2=J3=8 Hz, 1H); 2.11 (dd, J1=13.0 Hz， $\mathrm{J} 2=6.5 \mathrm{~Hz}, 1 \mathrm{H}) ; 2.04-1.94(\mathrm{~m}, 4 \mathrm{H}) ; 1.91-1.87(\mathrm{~m}, 1 \mathrm{H}) ; 1.84-1.76(\mathrm{~m}, 4 \mathrm{H}) ; 1.50$ ( $\mathrm{s}$, $3 \mathrm{H}) ; 1.38(\mathrm{~s}, 3 \mathrm{H}) ; 1.21(\mathrm{~s}, 9 \mathrm{H}) ; 1.12-1.09(\mathrm{~m}, 21 \mathrm{H}) ; 0.76-0.75(\mathrm{~m}, 6 \mathrm{H}) .{ }^{13} \mathrm{C} \operatorname{NMR}(75$ $\left.\mathrm{MHz}, \mathrm{C}_{6} \mathrm{D}_{6}\right) ; \delta(\mathrm{ppm}): 177.6,159.6,147.4,131.5,129.2,114.0,109.0,103.9,77.7$, $76.5,72.7,69.7,67.9,65.0,64.1,54.7,38.8,37.5,35.7,32.4,28.5,27.6,27.4$, $26.7,25.4,23.7,22.2,18.2,14.6,12.3,11.6$. HRMS (ESI) calcd for $\mathrm{C}_{42} \mathrm{H}_{72} \mathrm{O}_{8} \mathrm{SiNa}$ $[\mathrm{M}+\mathrm{Na}] 755.48941$, found 755.49282 .

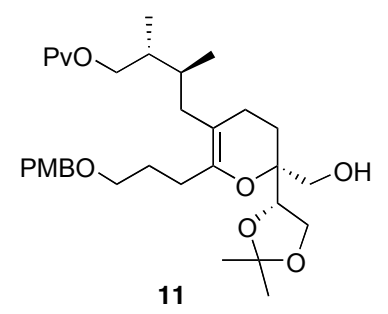

Alcohol 11. Tetra-n-butylammonium fluoride hydrate $(0.58 \mathrm{~g}, 1.84 \mathrm{mmol})$ was added to a solution of the substrate $(0.456 \mathrm{~g}, 0.622 \mathrm{mmol})$ in THF $(8.0 \mathrm{ml})$ and the mixture was stirred at room temperature for $0.5 \mathrm{~h}$. Saturated aqueous ammonium chloride was added, and the mixture was extracted with EtOAc, washed with water and brine, dried with anhydrous sodium sulfate, and concentrated under reduced pressure. The resultant oil was purified by column chromatography (silica, 40\% ethyl acetatehexanes containing $1.5 \%$ triethylamine) to give $0.325 \mathrm{~g}(0.563 \mathrm{mmol}$, 91\%) of the primary alcohol. $[\alpha]_{\mathrm{D}}^{20}+13.6^{\circ}\left(\mathrm{C} 1.0 \mathrm{CH}_{2} \mathrm{Cl}_{2}\right)$. IR (film, $\left.\mathrm{cm}^{-1}\right)$ : $3444,2960,2934$, $1728,1680,1614,1514,1480,1396,1284,1098,1038 .{ }^{1} \mathrm{H} \operatorname{NMR}\left(300 \mathrm{MHz}, \mathrm{C}_{6} \mathrm{D}_{6}\right) ; \delta(\mathrm{ppm})$ : $7.24(\mathrm{~d}, \mathrm{~J}=8.4 \mathrm{~Hz}, 2 \mathrm{H}) ; 6.82(\mathrm{~d}, \mathrm{~J}=8.4 \mathrm{~Hz}, 2 \mathrm{H}) ; 4.41$ (t, J=6.6 Hz, $1 \mathrm{H}) ; 4.32$ ( $2 \mathrm{H}) ; 4.10-3.85(\mathrm{~m}, 4 \mathrm{H}) ; 3.70-3.60(\mathrm{bm}, 2 \mathrm{H}) ; 3.33(\mathrm{~s}, 3 \mathrm{H}) ; 3.39-3.29(\mathrm{~m}, 2 \mathrm{H}) ; 2.45$ (bs, $1 \mathrm{H}) ; 2.28$ (t, J=7.2 Hz, 2H); $2.00-1.68(\mathrm{~m}, 10 \mathrm{H}) ; 1.41(\mathrm{~s}, 3 \mathrm{H}) ; 1.27$ (s, 3H) ; $1.20(\mathrm{~s}, 9 \mathrm{H}) ; 0.73(\mathrm{~d}, \mathrm{~J}=6.6 \mathrm{~Hz}, 3 \mathrm{H}) ; 0.69(\mathrm{~d}, \mathrm{~J}=6.6 \mathrm{~Hz}, 3 \mathrm{H}) .{ }^{13} \mathrm{C} \operatorname{NMR}\left(75 \mathrm{MHz}, \mathrm{C}_{6} \mathrm{D}_{6}\right)$; $\delta(\mathrm{ppm}): 177.9,159.7,146.9,131.1,129.5,114.2,114.0,109.2,104.4,78.7,76.2$, $72.8,69.7,68.1,65.0,63.2,54.8,38.9,37.3,35.8,32.4,28.2,27.7,27.4,26.4$, $25.1,22.5,21.8,14.7,11.8$. HRMS (ESI) calcd for $\mathrm{C}_{33} \mathrm{H}_{52} \mathrm{O}_{8} \mathrm{Na}$ [M+Na] 599.35599, found 599.35659 . 


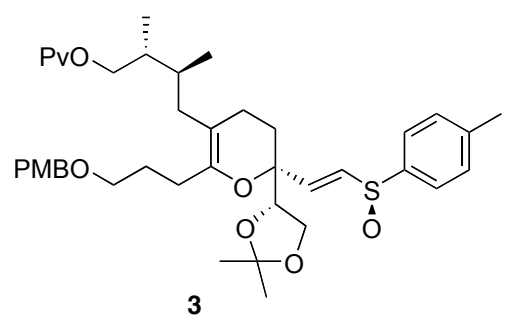

Vinyl sulfoxide 3 . Sulfur trioxide-pyridine complex $(0.23 \mathrm{~g}, 1.45 \mathrm{mmol})$ was added in one portion at $0^{\circ} \mathrm{C}$ to a solution of alcohol 11 (0.365 g, $\left.0.633 \mathrm{mmol}\right)$, DMSO (0.49 $\mathrm{ml}, 6.91 \mathrm{mmol})$, and triethylamine $(0.72 \mathrm{ml}, 5.16 \mathrm{mmol})$ in dry dichloromethane $(2.6$ $\mathrm{ml}$ ). The reactio mixture was stirred at room temperature for $12 \mathrm{~h}$, and then diluted with a 1:1 mixture of ethyl acetate-hexanes, and washed water several times. The combined aqueous layers were extracted with a 1:1 mixture of ethyl acetate-hexanes, and the combined organic layers were washed with brine, dried over anhydrous sodium sulfate and filtered through a $1.5 \mathrm{~cm}$ pad of silica pretreated with triethylamine. A 2\% triethylamine in a 1:1 mixture of EtOAc-hexanes was used as an eluent. The resultant solution was concentrated under reduced pressure and the residue was dried in vacuum to give the aldehyde (0.329 $\mathrm{g}, 0.572 \mathrm{mmol}, 90 \%)$, which was used directly in the next step.

To a solution of LDA $\left[i-\mathrm{Pr}_{2} \mathrm{NH}(0.17 \mathrm{ml}, 1.21 \mathrm{mmol}), n\right.$-BuLi $(2.38 \mathrm{M}$ in hexanes, 0.47 $\left.\mathrm{ml}, 1.10 \mathrm{mmol}), \operatorname{THF}(1.50 \mathrm{ml}), 0^{\circ} \mathrm{C}, 10 \mathrm{~min}\right]$ was added a solution of $S$-methyl p-tolyl sulfoxide $(0.152 \mathrm{~g}, 0.986 \mathrm{mmol})$ in THF $(0.70 \mathrm{ml}$ total with rinses $)$ at $0^{\circ} \mathrm{C}$ and the mixture was stirred for $20 \mathrm{~min}$ and cooled to $-78^{\circ} \mathrm{C}$. A solution of the aldehyde $(0.352 \mathrm{~g}, 0.612 \mathrm{mmol})$ in $\mathrm{THF}(2.0 \mathrm{ml}$ total with rinses $)$ was added, and the reaction mixture was stirred for $1 \mathrm{~h}$. The cooling bath was removed and the reaction mixture was quenched by addition of saturated aqueous ammonium chloride and extracted with EtOAc. The organic phase was dried with anhydrous sodium sulfate and concentrated. The residue was purified by column chromatography (silica, $40 \rightarrow 50 \%$ ethyl acetatehexanes with $\left.0.3 \% \mathrm{Et}_{3} \mathrm{~N}\right)$ to give $0.167 \mathrm{~g}(0.229 \mathrm{mmol}$, 37\%) of the less polar hydroxy sulfoxide (A) and $0.315 \mathrm{~g}(0.295 \mathrm{mmol}$, 48\%) of the more polar hydroxy sulfoxide (B). A : $[\alpha]_{\mathrm{D}}{ }^{20}-101.1^{\circ}\left(\mathrm{C} 1.0 \mathrm{CH}_{2} \mathrm{Cl}_{2}\right)$. IR (film, $\left.\mathrm{cm}^{-1}\right): 3356,2962,2934,2876,1728$, 1716, 1682, 1614, 1514, 1494, 1480, 1470, 1462, 1370, 1286, 1248, 1212, 1160, 1066, $1014,848 .{ }^{1} \mathrm{H} \operatorname{NMR}\left(500 \mathrm{MHz}, \mathrm{C}_{6} \mathrm{D}_{6}\right) ; \delta(\mathrm{ppm}): 7.43(\mathrm{~d}, \mathrm{~J}=8.0 \mathrm{~Hz}, 2 \mathrm{H}) ; 7.29(\mathrm{~d}, \mathrm{~J}=8.0 \mathrm{~Hz}$, $2 \mathrm{H}) ; 6.88(\mathrm{~d}, \mathrm{~J}=8.0 \mathrm{~Hz}, 2 \mathrm{H}) ; 6.84(\mathrm{~d}, \mathrm{~J}=8.0 \mathrm{~Hz}, 2 \mathrm{H}) ; 4.65(\mathrm{~m}, 1 \mathrm{H}) ; 4.41$ ( $\mathrm{AB}$ ， $\mathrm{J} 1=11.5 \mathrm{~Hz}, \mathrm{~J} 2=14.5 \mathrm{~Hz}, 2 \mathrm{H}) ; 4.11-4.06(\mathrm{~m}, 2 \mathrm{H}) ; 4.01-3.97(\mathrm{~m}, 3 \mathrm{H}) ; 3.75(\mathrm{dd}, \mathrm{J} 1=8.0$ $\mathrm{Hz}, \mathrm{J} 2=7.0 \mathrm{~Hz}, 1 \mathrm{H}) ; 3.49-3.44(\mathrm{~m}, 2 \mathrm{H}) ; 3.33(\mathrm{~s}, 3 \mathrm{H}) ; 2.95-2.92(\mathrm{~m}, 2 \mathrm{H}) ; 2.27-2.22$ $(\mathrm{m}, 2 \mathrm{H}) ; 2.18(\mathrm{ddd}, \mathrm{J} 1=\mathrm{J} 2=\mathrm{J} 3=7.0 \mathrm{~Hz}, 1 \mathrm{H}) ; 2.13-2.06(\mathrm{~m}, 1 \mathrm{H}) ; 1.98(\mathrm{~s}, 3 \mathrm{H}) ; 1.89-$ $1.68(\mathrm{~m}, 8 \mathrm{H}) ; 1.34(\mathrm{~s}, 3 \mathrm{H}) ; 1.26(\mathrm{~s}, 9 \mathrm{H}) ; 1.17(\mathrm{~s}, 3 \mathrm{H}) ; 0.80(\mathrm{~d}, \mathrm{~J}=6.5 \mathrm{~Hz}, 3 \mathrm{H})$; $0.72(\mathrm{~d}, \mathrm{~J}=7.0 \mathrm{~Hz}, 3 \mathrm{H}) .{ }^{13} \mathrm{C} \operatorname{NMR}\left(75 \mathrm{MHz}, \mathrm{C}_{6} \mathrm{D}_{6}\right) ; \delta(\mathrm{ppm}): 177.9,159.7,146.5,142.5$, $140.7,131.4,130.0,129.8,129.4,124.5,124.2,114.1,109.5,105.6,78.1,76.4$, 
$72.8,69.8,68.0,67.5,65.1,60.4,54.8,38.9,37.1,36.1,32.5,28.5,27.44$, $27.36,26.3,25.3,24.9,22.4,21.5,21.0,14.6,12.0$. HRMS (ESI) calcd for $\mathrm{C}_{41} \mathrm{H}_{60} \mathrm{O}_{9} \mathrm{SNa} \quad[\mathrm{M}+\mathrm{Na}] \quad 751.38557$, found 751.38518. B: $[\alpha]_{\mathrm{D}}{ }^{20}-57.1^{\circ}\left(\mathrm{C} 1.0 \mathrm{CH}_{2} \mathrm{Cl}_{2}\right)$. IR $\left(f i l m, \mathrm{~cm}^{-1}\right): 2960,2942,2866,1730,1614,1514,1462,1380,1284,1156,1098$, 1070, 1014, 882. ${ }^{1} \mathrm{H}$ NMR $\left(500 \mathrm{MHz}, \mathrm{C}_{6} \mathrm{D}_{6}\right) ; \delta(\mathrm{ppm}): 7.44(\mathrm{~d}, \mathrm{~J}=7.5 \mathrm{~Hz}, 2 \mathrm{H}) ; 7.24$ (d, $\mathrm{J}=8.5 \mathrm{~Hz}, 2 \mathrm{H}) ; 6.85-6.83(\mathrm{~m}, 4 \mathrm{H}) ; 4.77(\mathrm{bd}, \mathrm{J}=9.5 \mathrm{~Hz}, 1 \mathrm{H}) ; 4.63(\mathrm{dd}, \mathrm{J} 1=\mathrm{J} 2=7.5 \mathrm{~Hz}$ ， $1 \mathrm{H}) ; 4.32(\mathrm{~s}, 2 \mathrm{H}) ; 4.32-4.29(\mathrm{~m}, 1 \mathrm{H}) ; 4.18(\mathrm{dd}, \mathrm{J} 1=8.5 \mathrm{~Hz}, \mathrm{~J} 2=7.0 \mathrm{~Hz}, 1 \mathrm{H}) ; 4.08$ $(\mathrm{dd}, \mathrm{J} 1=11.0 \mathrm{~Hz}, \mathrm{~J} 2=7.0 \mathrm{~Hz}, 1 \mathrm{H}) ; 3.99(\mathrm{dd}, \mathrm{J} 1=8.5 \mathrm{~Hz}, \mathrm{~J} 2=6.5 \mathrm{~Hz}, 1 \mathrm{H}) ; 3.91$ (dd， $\mathrm{J} 1=11.0 \mathrm{~Hz}, \mathrm{~J} 2=7.0 \mathrm{~Hz}, 1 \mathrm{H}) ; 3.37-3.31(\mathrm{~m}, 2 \mathrm{H}) ; 3.34(\mathrm{~s}, 3 \mathrm{H}) ; 3.19$ ( $\mathrm{dd}, \mathrm{J} 1=14.0 \mathrm{~Hz}$, $\mathrm{J} 2=10.5 \mathrm{~Hz}, 1 \mathrm{H}) ; 3.11(\mathrm{dd}, \mathrm{J} 1=14.0 \mathrm{~Hz}, \mathrm{~J} 2=2.0 \mathrm{~Hz}, 1 \mathrm{H}) ; 2.27$ (t, J=7.5 Hz, 2H); 1.95 $(\mathrm{s}, 3 \mathrm{H}) ; 1.93-1.88(\mathrm{~m}, 2 \mathrm{H}) ; 1.84-1.64(\mathrm{~m}, 8 \mathrm{H}) ; 1.43(\mathrm{~s}, 3 \mathrm{H}) ; 1.29(\mathrm{~s}, 3 \mathrm{H}) ; 1.21$ ( $\mathrm{s}$, $9 \mathrm{H}) ; 0.77(\mathrm{~d}, \mathrm{~J}=7.5 \mathrm{~Hz}, 3 \mathrm{H}) ; 0.71(\mathrm{~d}, \mathrm{~J}=6.5 \mathrm{~Hz}, 3 \mathrm{H}) .{ }^{13} \mathrm{C} \operatorname{NMR}\left(75 \mathrm{MHz}, \mathrm{C}_{6} \mathrm{D}_{6}\right) ; \delta(\mathrm{ppm})$ : 177.7, 159.7, 146.7, 142.2, 141.1 131.1, 130.0, 129.5, 124.3, 114.0, 109.3, 105.2, $77.7,76.8,72.7,69.6,68.6,67.9,65.5,59.0,54.8,38.9,37.2,36.6,33.0,28.0$, $27.7,27.4,26.5,25.4,22.8,21.8,21.0,14.8,12.2$. HRMS (ESI) calcd for $\mathrm{C}_{41} \mathrm{H}_{60} \mathrm{O} 9 \mathrm{SNa}[\mathrm{M}+\mathrm{Na}] 751.38557$, found 751.38750 .

The diastereomeric hydroxy sulfoxides were combined and the mixture $(0.382 \mathrm{~g}, 0.524$ mmol) was dissolved in THF $(10 \mathrm{ml})$. The solution was cooled to $-78^{\circ} \mathrm{C}$ and $\operatorname{TMSCl}(0.33$ $\mathrm{ml}, 2.6 \mathrm{mmol})$ was added followed by KHMDS (0.5M in PhMe, $5.5 \mathrm{ml}, 2.75 \mathrm{mmol})$. After $15 \mathrm{~min}$, the reaction mixture was quenched by addition of saturated aqueous ammonium chloride and the aqueous phase was extracted with EtOAc. The combined organic layers were dried with anhydrous sodium sulfate and concentrated. The residue was dissolved in THF $(3.0 \mathrm{ml})$ and added to a solution of LDA $\left[i-\mathrm{Pr}_{2} \mathrm{NH}(0.24 \mathrm{ml}, 1.71\right.$ mmol), n-BuLi (2.38M in hexanes, $\left.0.66 \mathrm{mml}, 1.57 \mathrm{mmol}), \mathrm{THF}(2.00 \mathrm{ml}), 0^{\circ} \mathrm{C}, 10 \mathrm{~min}\right]$ at $-78^{\circ} \mathrm{C}$. After $30 \mathrm{~min}$, the mixture was quenched with saturated aqueous ammonium chloride and diluted with EtOAc. The organic phase was washed with water and the combined aqueous layers were extracted with ethyl acetate. The combined organic layers were washed with brine, dried with anhydrous sodium sulfate and concentrated. The residue was subjected to column chromatography (silica, 40 $\rightarrow 50 \%$ ethyl acetate-hexanes) to yield $0.313 \mathrm{~g}(0.440 \mathrm{mmol}, 84 \%$ of the requisite vinylic sulfoxide 3. $[\alpha]_{D}{ }^{20}-84.6^{\circ}\left(\mathrm{C} 1.0 \quad \mathrm{CH}_{2} \mathrm{Cl}_{2}\right)$. IR $\left(\mathrm{film}, \mathrm{cm}^{-1}\right): 2960,2934,1728,1714$, $1614,1504,1480,1370,1210,1160,1070,672 .{ }^{1} \mathrm{H} \operatorname{NMR}\left(500 \mathrm{MHz}, \mathrm{C}_{6} \mathrm{D}_{6}\right) ; \delta(\mathrm{ppm}): 7.42$ $(\mathrm{d}, \mathrm{J}=8.0 \mathrm{~Hz}, 2 \mathrm{H}) ; 7.23(\mathrm{~d}, \mathrm{~J}=8.5 \mathrm{~Hz}, 2 \mathrm{H}) ; 6.91(\mathrm{~d}, \mathrm{~J}=8.0 \mathrm{~Hz}, 2 \mathrm{H}) ; 6.82(\mathrm{~d}, \mathrm{~J}=8.5$ $\mathrm{Hz}, 2 \mathrm{H}) ; 6.59(\mathrm{~d}, \mathrm{~J}=15.0 \mathrm{~Hz}, 1 \mathrm{H}) ; 6.39(\mathrm{~d}, \mathrm{~J}=15.0 \mathrm{~Hz}, 1 \mathrm{H}) ; 4.29$ (AB, $2 \mathrm{H}) ; 4.02$ (dd, $\mathrm{J} 1=11.0 \mathrm{~Hz}, \mathrm{~J} 2=6.5 \mathrm{~Hz}, 1 \mathrm{H}) ; 3.95$ (dd，J1=J2=5.5 Hz, $1 \mathrm{H}) ; 3.91$ (dd, J1=J2=6.5 Hz， $1 \mathrm{H}) ; 3.89(\mathrm{dd}, \mathrm{J} 1=11.0 \mathrm{~Hz}, \mathrm{~J} 2=6.5 \mathrm{~Hz}, 1 \mathrm{H}) ; 3.73(\mathrm{dd}, \mathrm{J} 1=8.0 \mathrm{~Hz}, \mathrm{~J} 2=6.5 \mathrm{~Hz}, 1 \mathrm{H})$; $3.33(\mathrm{~s}, 3 \mathrm{H}) ; 3.31-3.25(\mathrm{~m}, 2 \mathrm{H}) ; 2.30-2.18(\mathrm{~m}, 2 \mathrm{H}) ; 2.00(\mathrm{~s}, 3 \mathrm{H}) ; 1.88-1.54(\mathrm{~m}$, $11 \mathrm{H}) ; 1.44(\mathrm{~s}, 3 \mathrm{H}) ; 1.28(\mathrm{~s}, 9 \mathrm{H}) ; 1.23(\mathrm{~s}, 9 \mathrm{H}) ; 0.71(\mathrm{~d}, \mathrm{~J}=7.0 \mathrm{~Hz}, 3 \mathrm{H}) ; 0.61$ (d, 
$\mathrm{J}=7.0 \mathrm{~Hz}, 3 \mathrm{H}) .{ }^{13} \mathrm{C} \operatorname{NMR}\left(75 \mathrm{MHz}, \mathrm{C}_{6} \mathrm{D}_{6}\right) ; \delta(\mathrm{ppm}): 177.6,159.7,146.8,142.5,141.0$, $138.2,136.4,131.3,130.1,129.3,124.6,114.0,110.0,105.6,80.1,77.8,72.7$, $69.4,67.7,64.8,54.8,38.9,36.8,36.4,32.9,28.3,27.4,27.3,26.4,25.3,22.0$, 21.0, 14.7, 12.0. HRMS (ESI) calcd for $\mathrm{C}_{41} \mathrm{H}_{58} \mathrm{O}_{8} \mathrm{SNa}$ [M+Na] 733.37501, found 733.37697.



Vinyl sulfoxide epi-3. This vinylic sulfoxide was prepared by the same method that was used for sulfoxide 3. $[\alpha]_{\mathrm{D}}{ }^{20}+45.1^{\circ}\left(\mathrm{C} 0.5 \mathrm{CH}_{2} \mathrm{Cl}_{2}\right)$. IR (film, cm$\left.{ }^{-1}\right): 2960,2932$, $1728,1680,1614,1514,1480,1462,1380,1302,1248,1210,1070,812 .{ }^{1} \mathrm{H}$ NMR $(500$ $\left.\mathrm{MHz}, \mathrm{C}_{6} \mathrm{D}_{6}\right) ; \delta(\mathrm{ppm}): 7.48(\mathrm{~d}, \mathrm{~J}=8.0 \mathrm{~Hz}, 2 \mathrm{H}) ; 7.23(\mathrm{~d}, \mathrm{~J}=8.5 \mathrm{~Hz}, 2 \mathrm{H}) ; 6.87(\mathrm{~d}, \mathrm{~J}=8.0$ $\mathrm{Hz}, 2 \mathrm{H}) ; 6.82(\mathrm{~d}, \mathrm{~J}=8.5 \mathrm{~Hz}, 2 \mathrm{H}) ; 6.62(\mathrm{~d}, \mathrm{~J}=15.0 \mathrm{~Hz}, 1 \mathrm{H}) ; 6.49$ (d, J=15.0 Hz, 1H)； $4.30(\mathrm{~s}, 2 \mathrm{H}) ; 4.07(\mathrm{dd}, \mathrm{J} 1=11.0 \mathrm{~Hz}, \mathrm{~J} 2=7.0 \mathrm{~Hz}, 1 \mathrm{H}) ; 3.95(\mathrm{dd}, \mathrm{J} 1=11.0 \mathrm{hz}, \mathrm{J} 2=7.0$ $\mathrm{Hz}, 1 \mathrm{H}) ; 3.91-3.87(\mathrm{~m}, 2 \mathrm{H}) ; 3.72(\mathrm{dd}, \mathrm{J} 1=10.5 \mathrm{~Hz}, \mathrm{~J} 2=9.0 \mathrm{~Hz}, 1 \mathrm{H}) ; 3.32(\mathrm{~s}, 3 \mathrm{H})$; $3.35-3.29(\mathrm{~m}, 2 \mathrm{H}) ; 2.34$ (ddd, J1=14.5 Hz，J2=J3=7.5 Hz, 1H); 2.22 (ddd, J1=14.5 Hz， $\mathrm{J} 2=\mathrm{J} 3=7.0 \mathrm{~Hz}, 1 \mathrm{H}) ; 1.98-1.92(\mathrm{~m}, 1 \mathrm{H}) ; 1.94(\mathrm{~s}, 3 \mathrm{H}) ; 1.89-1.82(\mathrm{~m}, 3 \mathrm{H}) ; 1.77-1.71$ $(\mathrm{m}, 3 \mathrm{H}) ; 1.67-1.57(\mathrm{~m}, 3 \mathrm{H}) ; 1.41(\mathrm{~s}, 3 \mathrm{H}) ; 1.26-1.19(\mathrm{~m}, 3 \mathrm{H}) ; 1.25(\mathrm{~s} ， 9 \mathrm{H}) ; 0.75(\mathrm{~d}$ ， $\mathrm{J}=7.0 \mathrm{~Hz}, 3 \mathrm{H}) ; 0.69(\mathrm{~d}, \mathrm{~J}=7.0 \mathrm{~Hz}, 3 \mathrm{H}) .{ }^{13} \mathrm{C} \mathrm{NMR}\left(75 \mathrm{MHz}, \mathrm{C}_{6} \mathrm{D}_{6}\right) ; \delta(\mathrm{ppm}): 177.7,159.7$, $146.8,142.5,141.0,138.2,136.0,131.3,130.2,129.3,124.7,114.0,110.0,105.6$, $80.0,78.0,72.7,69.4,67.8,64.8,54.8,38.9,37.2,36.1,32.7,28.3,27.5,27.3$, $26.4,25.3,22.1,21.0,14.8,11.9$. HRMS (ESI) calcd for $\mathrm{C}_{41} \mathrm{H}_{58} \mathrm{O}_{8} \mathrm{SNa}$ [M+Na] 733.37501 , found 733.37617 .



Azide 14. A solution of the pivaloate $3(78 \mathrm{mg}, 0.110 \mathrm{mmol})$ in dichloromethane (2.8 $\mathrm{ml}$ ) was cooled to $-78^{\circ} \mathrm{C}$ and DIBAL ( $1 \mathrm{M}$ in PhMe, $0.22 \mathrm{ml}, 0.22 \mathrm{mmol}$ ) was added. After $20 \mathrm{~min}$, the reaction was quenched by addition of saturated aqueous solution of Rochelle's salt, stirred at room temperature for $1 \mathrm{~h}$, and the aqueous phase was extracted with ethyl acetate $(2 \times 5 \mathrm{ml})$, the combined organic layers were washed with brine, dried with anhydrous sodium sulfate and concentrated to yield $70 \mathrm{mg}(0.110$ 
mmol, $100 \%$ of the requisite primary alcohol, which was used without further purification.

The alcohol (70 mg, $0.110 \mathrm{mmol})$ was dissolved in dichloromethane (0.50 ml) and treated with tosyl chloride (27 mg, $0.142 \mathrm{mmol})$, pyridine (0.10 ml, $1.23 \mathrm{mmol})$, and DMAP (2 mg, $0.016 \mathrm{mmol}$ ) at $50^{\circ} \mathrm{C}$ for $24 \mathrm{~h}$. Upon cooling to rt, the reaction mixture was diluted with ethyl acetate and washed with saturated aqueous ammonium chloride, water and brine, dried with anhydrous sodium sulfate and concentrated. The residue was subjected to column chromatography (silica, 50\% ethyl acetate-hexanes) to yield $51 \mathrm{mg}(0.065 \mathrm{mmol}, 59 \%)$ of the tosylate along with $5 \mathrm{mg} \mathrm{(0.008} \mathrm{mmol,} \mathrm{7 \% )} \mathrm{of} \mathrm{the}$ starting alcohol. Sodium azide $(27 \mathrm{mg}, 0.42 \mathrm{mmol})$ was added to a solution of the tosyate $(46 \mathrm{mg}, 0.059 \mathrm{mmol})$ in $\operatorname{DMF}(0.40 \mathrm{ml})$ and the mixture was stirred at $70^{\circ} \mathrm{C}$ for $3.5 \mathrm{~h}$. After cooling the mixtire was diluted with ethyl acetate and washed with water $(4 \times 4 \mathrm{ml})$, the combined aqueous layers were extracted with ethyl acetate (2x3 ml) and the combined organic layers were washed with brine, dried with anhydrous sodium sulfate and concentrated. Column chromatography of the residue (silica, 45\% ethyl acetate-hexanes) afforded $33 \mathrm{mg}(50.6 \mu \mathrm{mol}, 86 \%)$ of $\mathbf{1 4}^{4}[\boldsymbol{\alpha}]_{\mathrm{D}}^{20}-80.1^{\circ}(\mathrm{C} 0.5$ $\left.\mathrm{CH}_{2} \mathrm{Cl}_{2}\right)$. IR (film, $\left.\mathrm{cm}^{-1}\right): 2960,2934,2096,1678,1614,1514,1462,1456,1380,1248$, $1212,1174,1154,1070,1016,968,850,736 .{ }^{1} \mathrm{H} N M R \quad\left(500 \mathrm{MHz}, \mathrm{C}_{6} \mathrm{D}_{6}\right) ; \delta(\mathrm{ppm}): 7.45(\mathrm{~d}$, $\mathrm{J}=8.0 \mathrm{~Hz}, 2 \mathrm{H}) ; 7.23(\mathrm{~d}, \mathrm{~J}=8.0 \mathrm{~Hz}, 2 \mathrm{H}) ; 6.87(\mathrm{~d}, \mathrm{~J}=8.0 \mathrm{~Hz}, 2 \mathrm{H}) ; 6.83(\mathrm{~d}, \mathrm{~J}=8.5 \mathrm{~Hz}$, $2 \mathrm{H}) ; 6.51(\mathrm{~d}, \mathrm{~J}=15.0 \mathrm{~Hz}, 1 \mathrm{H}) ; 6.38(\mathrm{~d}, \mathrm{~J}=15.0 \mathrm{~Hz}, 1 \mathrm{H}) ; 4.30$ (s, $2 \mathrm{H}) ; 3.92$ (dd, $\mathrm{J} 1=8.5 \mathrm{~Hz}, \mathrm{~J} 2=6.0 \mathrm{~Hz}, 1 \mathrm{H}) ; 3.87(\mathrm{dd}, \mathrm{J} 1=\mathrm{J} 2=6.0 \mathrm{~Hz}, 1 \mathrm{H}) ; 3.71$ ( dd, J1=8.0 Hz, J2=6.5 $\mathrm{Hz}, 1 \mathrm{H}) ; 3.33(\mathrm{~s}, 3 \mathrm{H}) ; 3.33-3.28(\mathrm{~m}, 2 \mathrm{H}) ; 2.98$ ( $\mathrm{dd}, \mathrm{J} 1=12.5 \mathrm{~Hz}$, J2=6.5 Hz, $1 \mathrm{H})$; $2.78(\mathrm{dd}, \mathrm{J} 1=12.5 \mathrm{~Hz}, \mathrm{~J} 2=8.5 \mathrm{~Hz}, 1 \mathrm{H}) ; 2.32$ ( $\mathrm{ddd}, \mathrm{J} 1=14.5 \mathrm{~Hz}$, J2=J3=8.5 Hz, $1 \mathrm{H})$; 2.17 (ddd, J1=14.5 Hz, J2=J3=8.0 Hz, 1H); 1.95 (,$\quad 3 \mathrm{H}) ; 1.88-1.76(\mathrm{~m}, 5 \mathrm{H}) ; 1.65-$ $1.51(\mathrm{~m}, 5 \mathrm{H}) ; 1.43(\mathrm{~s}, 3 \mathrm{H}) ; 1.27(\mathrm{~s}, 3 \mathrm{H}) ; 0.64(\mathrm{~d}, \mathrm{~J}=7.0 \mathrm{~Hz}, 3 \mathrm{H}) ; 0.55(\mathrm{~d}, \mathrm{~J}=6.5$ $\mathrm{Hz}, \quad 3 \mathrm{H}) .{ }^{13} \mathrm{C} \operatorname{NMR}\left(75 \mathrm{MHz}, \mathrm{C}_{6} \mathrm{D}_{6}\right) ; \delta(\mathrm{ppm}): 163.8,150.8,146.8,144.9,142.6,141.5$, $135.4,134.1,133.3,128.7,118.1,114.1,109.7,84.2,81.9,76.8,73.5,68.8$, $59.8,58.8,41.1,40.2,36.5,32.4,31.4,30.5,30.3,29.4,26.1,25.1,18.2,16.3$. HRMS (ESI) calcd for $\mathrm{C}_{36} \mathrm{H}_{49} \mathrm{~N}_{3} \mathrm{O}_{6} \mathrm{SNa}$ [M+Na] 674.32398, found 674.32388 .



Alylic alcohol 12. A solution of vinylic sulfoxide 3 (0.154 9, 0.217 mmol), triethyl phosphite (0.18 ml, $1.05 \mathrm{mmol})$, and s-collidine (0.14 ml, 1.05 mmol) in $\mathrm{MeOCH}_{2} \mathrm{CH}_{2} \mathrm{OCH}_{2} \mathrm{CH}_{2} \mathrm{OH}(2.7 \mathrm{ml})$ was heated at $150^{\circ} \mathrm{C}$ for $15 \mathrm{~h}$ in a sealed vial under 
argon. After cooling, the reaction mixture was diluted with EtOAc and washed with saturated aqueous ammonium chloride, water three times, and the combined aqueous layers were extracted with ethyl acetate. The combined organic layers were washed with brine, dried with anhydrous sodium sulfate and concentrated. The residue was subjected to column chromatography (silica, 40\% ethyl acetate-hexanes) to yield $0.117 \mathrm{~g}(0.199 \mathrm{mmol}, 92 \%)$ of 12 after drying in high vacuum at $50^{\circ} \mathrm{C}$ to remove residual s-collidine. $[\alpha]_{\mathrm{D}}{ }^{20}-25.4^{\circ}\left(\mathrm{C} 0.5 \mathrm{CH}_{2} \mathrm{Cl}_{2}\right)$. IR (film, $\left.\mathrm{cm}^{-1}\right)$ : 2928, 2934, 1726, $1706,1612,1514,1458,1370,1284,1068,672 .{ }^{1} \mathrm{H} \operatorname{NMR}\left(500 \mathrm{MHz}, \mathrm{C}_{6} \mathrm{D}_{6}\right) ; \delta(\mathrm{ppm}): 7.23$ $(\mathrm{d}, \mathrm{J}=8.5 \mathrm{~Hz}, 2 \mathrm{H}) ; 6.83(\mathrm{~d}, \mathrm{~J}=8.5 \mathrm{~Hz}, 2 \mathrm{H}) ; 5.93(\mathrm{~d}, \mathrm{~J}=10.0 \mathrm{~Hz}, 1 \mathrm{H}) ; 5.54$ (d, J=10.0 $\mathrm{Hz}, 1 \mathrm{H}) ; 4.30(\mathrm{~s}, 2 \mathrm{H}) ; 3.97-3.92(\mathrm{~m}, 2 \mathrm{H}) ; 3.88(\mathrm{dd}, \mathrm{J} 1=\mathrm{J} 2=8.0 \mathrm{~Hz}, 1 \mathrm{H}) ; 3.84$ (dd, $\mathrm{J} 1=11.5 \mathrm{~Hz}, \mathrm{~J} 2=6.5 \mathrm{~Hz}, 1 \mathrm{H}) ; 3.79(\mathrm{dd}, \mathrm{J} 1=8.0 \mathrm{~Hz}, \mathrm{~J} 2=7.0 \mathrm{~Hz}, 1 \mathrm{H}) ; 3.36-3.29(\mathrm{~m}, 2 \mathrm{H})$; $3.32(\mathrm{~s}, 3 \mathrm{H}) ; 2.61(\mathrm{ddd}, \mathrm{J} 1=18.5 \mathrm{~Hz}, \mathrm{~J} 2=\mathrm{J} 3=7.0 \mathrm{~Hz}, 1 \mathrm{H}) ; 2.48$ (ddd, J1=18.5 Hz, $\mathrm{J} 2=\mathrm{J} 3=7.0 \mathrm{~Hz}, 1 \mathrm{H}) ; 2.26(\mathrm{ddd}, \mathrm{J} 1=13.5 \mathrm{~Hz}, \mathrm{~J} 2=9.5 \mathrm{~Hz}, \mathrm{~J} 3=4.0 \mathrm{~Hz}, 1 \mathrm{H}) ; 2.04$ (bs, 1H); 1.97-1.92 (m，2H); 1.78-1.62 (m，5H); $1.40-1.35(\mathrm{~m} ， 1 \mathrm{H}) ; 1.35$ ( $\mathrm{s}, 3 \mathrm{H}) ; 1.29$ (dd， $\mathrm{J} 1=14.0 \mathrm{~Hz}, \mathrm{~J} 2=7.0 \mathrm{~Hz}, 1 \mathrm{H}) ; 1.27(\mathrm{~s}, 3 \mathrm{H}) ; 1.21 \mathrm{(s}, 9 \mathrm{H}) ; 0.68$ (d, J=7.0 Hz, 3H); $0.65(\mathrm{~d}, \mathrm{~J}=6.5 \mathrm{~Hz}, 3 \mathrm{H}) .{ }^{13} \mathrm{C} \operatorname{NMR}\left(75 \mathrm{MHz}, \mathrm{C}_{6} \mathrm{D}_{6}\right) ; \delta(\mathrm{ppm}): 210.7,177.7,159.7,135.0$, 131.2, 130.1, 129.4, 114.0, 109.4, 80.7, 72.6, 69.3, 69.0, 67.4, 65.2, 54.7, 52.8, $42.7,38.8,37.6,35.6,30.4,29.9,27.4,26.4,25.3,24.5,15.9,11.2$. HRMS (ESI) calcd for $\mathrm{C}_{34} \mathrm{H}_{52} \mathrm{O}_{8} \mathrm{SNa}[\mathrm{M}+\mathrm{Na}] 611.35599$, found 611.35310 .

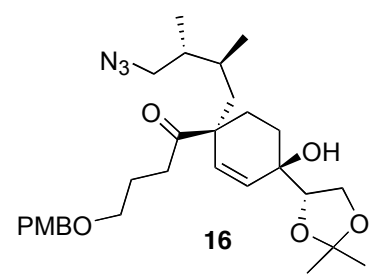

Azide 16. To a solution of pivaloate $12(0.117 \mathrm{~g}, 0.199 \mathrm{mmol})$ in dry methanol (4.0 ml) was added MeONa (2.4M in $\mathrm{MeOH}, 0.80 \mathrm{ml})$ and the mixture was stirred at $50^{\circ} \mathrm{C}$ for $15 \mathrm{~h}$ under argon. After cooling to room temperature, saturated aqueous ammonium chloride and dichloromethane were added. The aqueous phase was extracted with dichloromethane and the combined organic layers were dried with anhydrous sodium sulfate and concentrated. The residue was subjected to column chromatography (silica, $80 \%$ ethyl acetate-hexanes) to furnish $85 \mathrm{mg}(0.168 \mathrm{mmol}$, 85\%) of the primary alcohol along with $5 \mathrm{mg}(4 \%)$ of the starting material. $[\alpha]_{\mathrm{D}}{ }^{20}-21.5^{\circ}$ (c 0.31 $\left.\mathrm{CH}_{2} \mathrm{Cl}_{2}\right)$. IR (film, $\left.\mathrm{cm}^{-1}\right): 3390,2924,1614,1514,1470,1454,672 .{ }^{1} \mathrm{H}$ NMR $(500 \mathrm{MHz}$, $\left.\mathrm{CDCl}_{3}\right) ; \delta(\mathrm{ppm}): 7.24(\mathrm{~d}, \mathrm{~J}=9.0 \mathrm{~Hz}, 2 \mathrm{H}) ; 6.87(\mathrm{~d}, \mathrm{~J}=9.0 \mathrm{~Hz}, 2 \mathrm{H}) ; 6.10(\mathrm{~d}, \mathrm{~J}=10.0 \mathrm{~Hz}$, $1 \mathrm{H}) ; 5.64(\mathrm{~d}, \mathrm{~J}=10.0 \mathrm{~Hz}, 1 \mathrm{H}) ; 4.40$ (, $2 \mathrm{H}) ; 4.06(\mathrm{dd}, \mathrm{J} 1=\mathrm{J} 2=7.0 \mathrm{~Hz}, 1 \mathrm{H}) ; 3.99$ (dd, $\mathrm{J} 1=\mathrm{J} 2=8.0 \mathrm{~Hz}, 1 \mathrm{H}) ; 3.85(\mathrm{dd}, \mathrm{J} 1=\mathrm{J} 2=7.5 \mathrm{~Hz}, 1 \mathrm{H}) ; 3.80(\mathrm{~s}, 3 \mathrm{H}) ; 3.48-3.39(\mathrm{~m}, 4 \mathrm{H})$; $2.67(\mathrm{ddd}, \mathrm{J} 1=18.5 \mathrm{~Hz}, \mathrm{~J} 2=\mathrm{J} 3=7.0 \mathrm{~Hz}, 1 \mathrm{H}) ; 2.58(\mathrm{ddd}, \mathrm{J} 1=18.5 \mathrm{~Hz}, \mathrm{~J} 2=\mathrm{J} 3=7.0 \mathrm{~Hz}, 1 \mathrm{H})$; 
$2.22(\mathrm{~s}, 1 \mathrm{H}) ; 2.16(\mathrm{ddd}, \mathrm{J} 1=13.0 \mathrm{~Hz}, \mathrm{~J} 2=9.0 \mathrm{~Hz}, \mathrm{~J} 3=3.0 \mathrm{~Hz}, 1 \mathrm{H}) ; 1.85(\mathrm{p}, \mathrm{J}=6.5 \mathrm{~Hz}$, $2 \mathrm{H}) ; 1.81-1.69(\mathrm{~m}, 3 \mathrm{H}) ; 1.66-1.56(\mathrm{~m}, 2 \mathrm{H}) ; 1.52-1.45(\mathrm{~m}, 2 \mathrm{H}) ; 1.45(\mathrm{~s}, 3 \mathrm{H}) ; 1.40$ $(\mathrm{dd}, \mathrm{J} 1=13.5 \mathrm{~Hz}, \mathrm{~J} 2=6.0 \mathrm{~Hz}, 1 \mathrm{H}) ; 1.38(\mathrm{~s}, 3 \mathrm{H}) ; 0.75(\mathrm{~d}, \mathrm{~J}=6.5 \mathrm{~Hz}, 3 \mathrm{H}) ; 0.72(\mathrm{~d}$ ， $\mathrm{J}=6.5 \mathrm{~Hz}, 3 \mathrm{H}) .{ }^{13} \mathrm{C} \operatorname{NMR}\left(75 \mathrm{MHz}, \mathrm{CDCl}_{3}\right) ; \delta(\mathrm{ppm}): 212.6,159.4,135.5,130.7,129.6$, 114.0, 109.7, 80.3, 77.4, 72.7, 69.5, 69.1, 66.5, 65.3, 55.5, 53.1, 43.5, 41.2, 35.9, 30.4, 29.3, 27.7, 26.5, 25.4, 24.0, 16.2, 11.4. HRMS (ESI) calcd for $\mathrm{C}_{29} \mathrm{H}_{43} \mathrm{~N}_{3} \mathrm{O}_{6} \mathrm{Na} \quad[\mathrm{M}+\mathrm{Na}] 527.29847$, found 527.29905.

Triethylamine $(0.23 \mathrm{ml}, 1.6 \mathrm{mmol})$ was added to a solution of the primary alcohol (82 $\mathrm{mg}, 0.162 \mathrm{mmol})$ in dichloromethane $(0.80 \mathrm{ml})$, followed by $\mathrm{Ts}_{2} \mathrm{O}(80 \mathrm{mg}, 0.25$ mol) at room temperature. The mixture was stirred under argon for $20 \mathrm{~min}$, and then water $(0.10 \mathrm{ml})$ was added, the mixture was stirred for $10 \mathrm{~min}$, then diluted with dichloromethane and washed with water. The aqueous layers were extracted with dichloromethane, and the combined organic layers were washed with saturated aqueous $\mathrm{NaHCO}_{3}$, dried with anhydrous sodium sulfate and concentrated. The crude tosylate was dissolved in DMF (1.0 $\mathrm{ml})$ and sodium azide was added $(78 \mathrm{mg}, 1.20 \mathrm{mmol})$. The mixture was stirred at $70^{\circ} \mathrm{C}$ for $4 \mathrm{~h}$. After cooling to rt, the reaction mixture was diluted with ethyl acetate and washed with water $(4 \times 3 \mathrm{ml})$. The combined aqueous layers were extracted with ethyl acetate $(2 \times 3 \mathrm{ml})$ and the combined organic layers were washed with brine, dried with anhydrous sodium sulfate and concentrated. Column chromatography of the residue (silica, 40\% ethyl acetate-hexanes) afforded $81 \mathrm{mg}(0.153 \mathrm{mmol}, 94 \%)$ of the azide 16. $[\alpha]_{\mathrm{D}}{ }^{20}-19.1^{\circ}$ ( $\mathrm{C} 0.3 \mathrm{CH}_{2} \mathrm{Cl}_{2}$ ). IR (film, $\mathrm{cm}^{-}$ $\left.{ }^{1}\right): 3646,3626,2922,2098,1714,1652,1614,1470,1454 .{ }^{1} \mathrm{H} \operatorname{NMR}\left(500 \mathrm{MHz}, \mathrm{CDCl}_{3}\right)$; $\delta(\mathrm{ppm}): 7.24(\mathrm{~d}, \mathrm{~J}=9.0 \mathrm{~Hz}, 2 \mathrm{H}) ; 6.87(\mathrm{~d}, \mathrm{~J}=9.0 \mathrm{~Hz}, 2 \mathrm{H}) ; 6.40(\mathrm{~d}, \mathrm{~J}=10.5 \mathrm{~Hz}, 1 \mathrm{H})$; $5.65(\mathrm{~d}, J=10.5 \mathrm{~Hz}, 1 \mathrm{H}) ; 4.40(\mathrm{~s}, 2 \mathrm{H}) ; 4.07(\mathrm{dd}, \mathrm{J} 1=\mathrm{J} 2=7.0 \mathrm{~Hz}, 1 \mathrm{H}) ; 4.00$ ( $d d d$, $\mathrm{J} 1=8.0 \mathrm{~Hz}, \mathrm{~J} 2=6.5 \mathrm{~Hz}, 1 \mathrm{H}) ; 3.86(\mathrm{dd}, \mathrm{J} 1=\mathrm{J} 2=8.0 \mathrm{~Hz}, 1 \mathrm{H}) ; 3.80(\mathrm{~s}, 3 \mathrm{H}) ; 3.48-3.40(\mathrm{~m}$, $2 \mathrm{H}) ; 3.15(\mathrm{dd}, \mathrm{J} 1=12.5 \mathrm{~Hz}, \mathrm{~J} 2=7.5 \mathrm{~Hz}, 1 \mathrm{H}) ; 3.10(\mathrm{dd}, \mathrm{J} 1=12.5 \mathrm{~Hz}, \mathrm{~J} 2=7.0 \mathrm{~Hz}, 1 \mathrm{H})$; $2.65(\mathrm{ddd}, \mathrm{J} 1=18.0 \mathrm{~Hz}, \mathrm{~J} 2=\mathrm{J} 3=7.0 \mathrm{~Hz}, 1 \mathrm{H}) ; 2.57$ (ddd, J1=18.0 Hz, J2=J3=7.0 Hz, 1H); $2.23(\mathrm{~s}, 1 \mathrm{H}) ; 2.18(\mathrm{ddd}, \mathrm{J} 1=13.0 \mathrm{~Hz}, \mathrm{~J} 2=9.5 \mathrm{~Hz}, \mathrm{~J} 3=3.5 \mathrm{~Hz}, 1 \mathrm{H}) ; 1.85(\mathrm{p}, \mathrm{J}=6.0 \mathrm{~Hz}$, $2 \mathrm{H}) ; 1.78(\mathrm{ddd}, \mathrm{J} 1=13.0 \mathrm{~Hz}, \mathrm{~J} 2=9.5 \mathrm{~Hz}, \mathrm{~J} 3=3.5 \mathrm{~Hz}, 1 \mathrm{H}) ; 1.71-1.60(\mathrm{~m}, 4 \mathrm{H}) ; 1.50-1.45$ $(\mathrm{m}, 1 \mathrm{H}) ; 1.46(\mathrm{~s}, 3 \mathrm{H}) ; 1.41(\mathrm{dd}, \mathrm{J} 1=14.0 \mathrm{~Hz}, \mathrm{~J} 2=6.5 \mathrm{~Hz}, 1 \mathrm{H}) ; 1.38$ (s, 3H); 0.80 (d, $\mathrm{J}=7.0 \mathrm{~Hz}, 3 \mathrm{H}) ; 0.72(\mathrm{~d}, \mathrm{~J}=6.5 \mathrm{~Hz}, 3 \mathrm{H}) .{ }^{13} \mathrm{C} \mathrm{NMR}\left(75 \mathrm{MHz}, \mathrm{CDCl}_{3}\right) ; \delta(\mathrm{ppm}): 212.0,159.4$, 135.3, 130.8, 129.9, 129.5, 114.0, 109.7, 80.1, 72.7, 69.5, 69.1, 65.2, 56.0, 55.5, $53.0,43.1,38.5,35.9,30.5,30.3,27.2,26.5,25.4,24.1,15.8,12.3$. HRMS (ESI) calcd for $\mathrm{C}_{29} \mathrm{H}_{43} \mathrm{~N}_{3} \mathrm{O}_{6} \mathrm{Na}[\mathrm{M}+\mathrm{Na}] 552.30495$, found 552.30725 . 


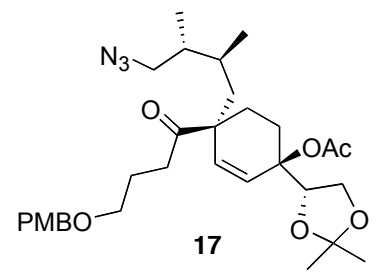

Allylic acetate 17. A solution of 16 (81 mg, $0.153 \mathrm{mmol}$, triethylamine (0.12 ml, $0.86 \mathrm{mmol})$, acetic anhydride (58 $\mu \mathrm{l}, 0.61 \mathrm{mmol})$, and DMAP (19 mg, $0.156 \mathrm{mmol})$ in dry dichloromethane $(0.80 \mathrm{ml})$ was refluxed at for $4.5 \mathrm{~h}$. The reaction mixture was quenched with $\mathrm{MeOH}(0.05 \mathrm{ml})$, diluted with dichloromethane and washed with 1M aqueous $\mathrm{HCl}$ and water, the combined aqueous layers were extracted with dichloromethane, and the combined organic phases were washed with saturated aqueous sodium bicarbonate, dried with anhydrous sodium sulfate and concentrated. Column chromatography of the residue (silica, 30\% ethyl acetate-hexanes) afforded 82 mg (0.143 mmol, 94\%) of 17. $[\alpha]_{\mathrm{D}}^{20}+10.4^{\circ}\left(\mathrm{c} 0.5 \mathrm{CH}_{2} \mathrm{Cl}_{2}\right)$. IR (film, $\left.\mathrm{cm}^{-1}\right): 2960,2934$, 2098, 1738, 1732, 1704, 1614, 1514, 1462, 1456, 1370, 1248, 1158, 1074, 1036. ${ }^{\mathrm{H}} \mathrm{NMR}$ $\left(500 \mathrm{MHz}, \mathrm{CDCl}_{3}\right) ; \delta(\mathrm{ppm}): 7.24(\mathrm{~d}, \mathrm{~J}=8.5 \mathrm{~Hz}, 2 \mathrm{H}) ; 6.87$ (d, J=8.5 Hz, 2H); $6.06(\mathrm{~d}$, $\mathrm{J}=10.5 \mathrm{~Hz}, 1 \mathrm{H}) ; 5.91(\mathrm{~d}, \mathrm{~J}=10.5 \mathrm{~Hz}, 1 \mathrm{H}) ; 4.52$ (dd, J1=J2=6.5 Hz, $1 \mathrm{H}) ; 4.40$ ( $\mathrm{s}, 2 \mathrm{H})$; 4.00 (dd, J1=8.5 Hz, J2=7.0 Hz, 1H); 3.92 (dd, J1=8.5 Hz, J2=6.5 Hz, 1H); 3.80 ( $3 \mathrm{H}) ; 3.49-3.41(\mathrm{~m}, 2 \mathrm{H}) ; 3.14(\mathrm{dd}, \mathrm{J} 1=12.5 \mathrm{~Hz}, \mathrm{~J} 2=7.5 \mathrm{~Hz}, 1 \mathrm{H}) ; 3.09$ (dd, J1=12.5 Hz, $\mathrm{J} 2=7.0 \mathrm{~Hz}, 1 \mathrm{H}) ; 2.70$ (ddd, J1=18.5 Hz, J2=7.5 Hz, J3=6.5 Hz, 1H); 2.57 (ddd, $\mathrm{J} 1=18.5 \mathrm{~Hz}, \mathrm{~J} 2=8.0 \mathrm{~Hz}, \mathrm{~J} 3=7.5 \mathrm{~Hz}, 1 \mathrm{H}) ; 2.18$ (ddd, J1=12.5 Hz, J2=8.5 Hz, J3=4.0 Hz, $1 \mathrm{H}) ; 2.09$ (ddd, J1=14.0 Hz, J2=9.5 Hz, J3=4.0 Hz, 1H); 1.96-1.84 (m, 3H); 1.95 ( $\mathrm{s}$, $3 \mathrm{H}) ; 1.70-1.60(\mathrm{~m}, 3 \mathrm{H}) ; 1.54$ (ddd, J1=13.5 Hz, J2=10.0 Hz, J3=3.5 Hz, $1 \mathrm{H}) ; 1.45$ ( $3 \mathrm{H}) ; 1.41(\mathrm{dd}, \mathrm{J} 1=13.5 \mathrm{~Hz}, \mathrm{~J} 2=6.0 \mathrm{~Hz}, 1 \mathrm{H}) ; 1.36(\mathrm{~s}, 3 \mathrm{H}) ; 0.81$ (d, J=6.5 Hz, $3 \mathrm{H})$; $0.72(\mathrm{~d}, \mathrm{~J}=6.5 \mathrm{~Hz}, 3 \mathrm{H}) .{ }^{13} \mathrm{C} \mathrm{NMR}\left(75 \mathrm{MHz}, \mathrm{CDCl}_{3}\right) ; \delta(\mathrm{ppm}): 211.9,170.0,159.4,135.1$, 130.9, 129.5, 129.0, 114.0, 110.0, 79.6, 78.0, 72.7, 69.3, 65.1, 56.0,55.5, 52.6, $43.2,38.6,35.9,30.2,27.2,26.2,25.2,24.9,24.0,22.2,15.9,12.3$. HRMS (ESI) calcd for $\mathrm{C}_{31} \mathrm{H}_{45} \mathrm{~N}_{3} \mathrm{O}_{7} \mathrm{Na}$ [M+Na] 594.31552, found 594.31279.



Spirobicyclic Imine 2. A solution of trimethylphosphine (1.0 M in THF, $0.11 \mathrm{ml}$ $0.11 \mathrm{mmol})$ was added to a solution of azide 17 (12.0 mg, 21 umol) in dry PhMe (0.14 ml) and the reaction mixture was stirred under argon for 5 h. Dry toluene (1.0 ml) was added and the solution was brought to reflux for 12 h. The solvent was 
evaporated under reduced pressure and the residue was dried in vacuum to give the cyclic imine $(11.5 \mathrm{mg})$ with $\sim 10 \mathrm{~mol} \% \mathrm{Me}_{3} \mathrm{PO}$ as the only impurity detected by $500 \mathrm{MHz}$ ${ }^{1} \mathrm{H}$ NMR. The imine was then purified by column chromatography (alumina, basic, activity $\mathrm{I}, 1 \% \mathrm{Et}_{3} \mathrm{~N}, 19 \%$ ethyl acetate-hexanes) to give pure 2 free of the phosphine oxide $(5.7 \mathrm{mg}, 10.8 \mu \mathrm{mol}, 51 \%) .[\alpha]_{\mathrm{D}}{ }^{20}+88.1^{\circ}\left(\mathrm{C} 0.5 \mathrm{CH}_{2} \mathrm{Cl}_{2}\right)$. IR (film, $\left.\mathrm{cm}^{-1}\right): 2958$, 2926, 1738, 1732, 1645, 1514, 1370, 1248, 1070, 1038. ${ }^{1} \mathrm{H} \operatorname{NMR}\left(500 \mathrm{MHz}, \mathrm{CDCl}_{3}\right)$; $\delta(\mathrm{ppm}): 7.23(\mathrm{~d}, \mathrm{~J}=8.0 \mathrm{~Hz}, 2 \mathrm{H}) ; 6.85(\mathrm{~d}, \mathrm{~J}=8.0 \mathrm{~Hz}, 2 \mathrm{H}) ; 6.09(\mathrm{~d}, \mathrm{~J}=10.5 \mathrm{~Hz}, 1 \mathrm{H})$; $6.05(\mathrm{~d}, \mathrm{~J}=10.5 \mathrm{~Hz}, 1 \mathrm{H}) ; 4.87(\mathrm{dd}, \mathrm{J} 1=7.0 \mathrm{~Hz}, \mathrm{~J} 2=6.0 \mathrm{~Hz}, 1 \mathrm{H}) ; 4.39$ (AB, J1=16.0 Hz, $\mathrm{J} 2=11.0 \mathrm{~Hz}, 2 \mathrm{H}) ; 3.98(\mathrm{dd}, \mathrm{J} 1=8.5 \mathrm{~Hz}, \mathrm{~J} 2=7.0 \mathrm{~Hz}, 1 \mathrm{H}) ; 3.89(\mathrm{dd}, \mathrm{J} 1=8.5 \mathrm{~Hz}, \mathrm{~J} 2=6.0$ $\mathrm{Hz}, 1 \mathrm{H}) ; 3.79(\mathrm{~s}, 3 \mathrm{H}) ; 3.75(\mathrm{dd}, \mathrm{J} 1=12.0 \mathrm{~Hz}, \mathrm{~J} 2=5.0 \mathrm{~Hz}, 1 \mathrm{H}) ; 3.54$ (dd, J1=12.0 Hz, $\mathrm{J} 2=2.0 \mathrm{~Hz}, 1 \mathrm{H}) ; 3.42(\mathrm{t}, \mathrm{J}=6.5 \mathrm{~Hz}, 2 \mathrm{H}) ; 2.22-2.14(\mathrm{~m}, 2 \mathrm{H}) ; 1.98-1.91(\mathrm{~m}, 2 \mathrm{H}) ; 1.91$ $(\mathrm{s}, 3 \mathrm{H}) ; 1.88-1.75(\mathrm{~m}, 5 \mathrm{H}) ; 1.49(\mathrm{bd}, \mathrm{J}=14.2 \mathrm{~Hz}, 1 \mathrm{H}) ; 1.45(\mathrm{~s}, 3 \mathrm{H}) ; 1.35-1.32$ (m, $1 \mathrm{H}) ; 1.34(\mathrm{~s}, 3 \mathrm{H}) ; 1.25-1.18(\mathrm{~m}, 1 \mathrm{H}) ; 1.04(\mathrm{~d}, \mathrm{~J}=7.5 \mathrm{~Hz}, 3 \mathrm{H}) ; 0.91(\mathrm{~d}, \mathrm{~J}=6.5 \mathrm{~Hz}$, $3 \mathrm{H}) \cdot{ }^{13} \mathrm{C} \operatorname{NMR}\left(100 \mathrm{MHz}, \mathrm{CDCl}_{3}\right) ; \delta(\mathrm{ppm}): 174.6,170.4,159.3,139.5,131.1,129.4$, 123.6, 113.9, 110.1, 79.0, 77.2, 72.6, 69.9, 65.1, 55.5, 53.2, 49.1, 45.1, 40.9, $34.4,34.2,28.2,27.7,26.2,24.7,23.8,21.9,21.7,18.8$. HRMS (ESI) calcd for $\mathrm{C}_{31} \mathrm{H}_{46} \mathrm{NO}_{6}[\mathrm{M}+\mathrm{H}] \quad 528.33251$, found 528.33151 .

Allylic alcohol B was prepared by the same method during preliminary model studies. The relative configuration of the new chiral centers in $\mathbf{B}$ was confirmed by HMQC, HMBC and NOE correlations:

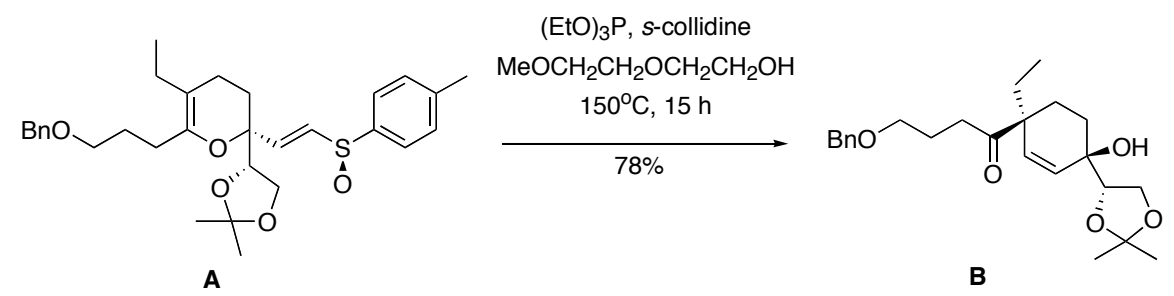

Allylic alcohol B. A solution of vinylic sulfoxide A (16.5 mg, 31.4 umol), triethyl phosphite (27 $\mu \mathrm{l}, 0.157 \mathrm{mmol})$, and s-collidine $(21 \mu \mathrm{l}, 0.16 \mathrm{mmol})$ in $\mathrm{MeOCH}_{2} \mathrm{CH}_{2} \mathrm{OCH}_{2} \mathrm{CH}_{2} \mathrm{OH}(0.25 \mathrm{ml})$ was heated at $150^{\circ} \mathrm{C}$ for $15 \mathrm{~h}$ in a sealed vial under argon. After cooling, the reaction mixture was diluted with EtOAc and washed with saturated aqueous ammonium chloride, water, and the combined aqueous layers were extracted with ethyl acetate. The combined organic layers were washed with brine, dried with anhydrous sodium sulfate and concentrated. The residue was subjected to column chromatography (silica, 30 $\rightarrow 50 \%$ ethyl acetate-hexanes) to yield $9.9 \mathrm{mg}$ (24.6 umol, $78 \%)$ of B. $[\alpha]_{\mathrm{D}}{ }^{20}-48.1^{\circ}\left(\mathrm{C} 0.5 \mathrm{CH}_{2} \mathrm{Cl}_{2}\right)$. IR $\left(\mathrm{film}, \mathrm{cm}^{-1}\right): 3484,2962,2360$, $1704,1372,1258,1212,1158,1096,1028,966 .{ }^{1} \mathrm{H}$ NMR $\left(500 \mathrm{MHz}, \mathrm{C}_{6} \mathrm{D}_{6}\right) ; \delta(\mathrm{ppm}): \mathrm{d}$ $(7.28(\mathrm{~d}, \mathrm{~J}=7.5 \mathrm{~Hz}, 2 \mathrm{H}) ; 7.19$ ( $t, J=7.5 \mathrm{~Hz}, 2 \mathrm{H}) ; 7.10$ (t, J=7.5 Hz, $1 \mathrm{H}) ; 5.76$ (d, $\mathrm{J}=10.0 \mathrm{~Hz}, 1 \mathrm{H}) ; 5.49(\mathrm{~d}, \mathrm{~J}=10.0 \mathrm{~Hz}, 1 \mathrm{H}) ; 4.28(\mathrm{~s}, 2 \mathrm{H}) ; 3.92-3.87(\mathrm{~m}, 2 \mathrm{H}) ; 3.75$ (dd, 
$\mathrm{J} 1=\mathrm{J} 2=5.5 \mathrm{~Hz}, 1 \mathrm{H}) ; 3.32-3.24(\mathrm{~m}, 2 \mathrm{H}) ; 2.46$ ( ddd, J1=17.5 Hz, J2=J3=7.0 Hz, $1 \mathrm{H})$; 2.37 (ddd, J1=17.5 Hz, J2=J3=7.0 Hz, 1H); 2.09 (bs, 1H); 1.94-1.87 (m, 2H); 1.79 (ddd, J1=13.5 Hz, J2=8.5 Hz, J3=3.5 Hz, 1H); 1.69 (ddd, J1=13.5 Hz, J2=9.5 Hz, $\mathrm{J} 3=3.5 \mathrm{~Hz}, 1 \mathrm{H}) ; 1.39(\mathrm{q}, \mathrm{J}=7.5 \mathrm{~Hz}, 2 \mathrm{H}) ; 1.34(\mathrm{~s}, 3 \mathrm{H}) ; 1.31$ (m, $1 \mathrm{H}) ; 1.28$ ( $\mathrm{s}, 3 \mathrm{H})$; 0.66 ( $t, J=7.5 \mathrm{~Hz}, 3 \mathrm{H}) .{ }^{13} \mathrm{C} \mathrm{NMR}\left(75 \mathrm{MHz}, \mathrm{C}_{6} \mathrm{D}_{6}\right) ; \delta(\mathrm{ppm}): 210.6,139.3,134.6,130.6$, $128.5,127.6,109.3,80.4,72.9,69.5,69.4,65.3,53.1,35.4,31.0,30.9,30.2$, $26.6,26.4,25.5,24.4,8.9$. HRMS (ESI) calcd for $\mathrm{C}_{24} \mathrm{H}_{34} \mathrm{O}_{5} \mathrm{Na}$ [M+Na] 425.23039, found 425.23121 .

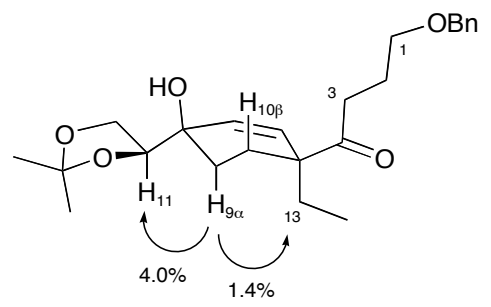

Table 1. Selected NOE for model allylic alcohol B.

\begin{tabular}{ccc}
\hline Proton (ppm) & NOE observed (\%) \\
\hline $\mathrm{H}_{10 \beta}(2.28)$ & $\mathrm{H}_{9 \beta}(2.3)$ \\
$\mathrm{H}_{9 \beta}(1.79)$ & $\mathrm{H}_{10 \beta}(2.9), \mathrm{H}_{11}(1.5)$ \\
$\mathrm{H}_{9 \alpha}(1.69)$ & $\mathrm{H}_{11}(4.0), \mathrm{H}_{13}(1.4)$ \\
$\mathrm{H}_{13}(1.39)$ & $\mathrm{H}_{9 \alpha}(0.9), \mathrm{H}_{3}(0.4,1.0)$ \\
\hline
\end{tabular}

